\title{
Hsp90-Induced Evolution: Adaptationist, Neutralist, and Developmentalist Scenarios
}

ROBERTA L. MILLSTEIN

Department of Philosophy

University of California, Davis

One Shields Avenue

Davis, CA 95616

USA

E-mail: RLMillstein@UCDavis.edu

Forthcoming in Biological Theory - published version may differ slightly from this version.

\section{Abstract}

Recent work on the heat-shock protein Hsp90 by Rutherford and Lindquist (1998) has been included among the pieces of evidence taken to show the essential role of developmental processes in evolution; Hsp90 acts as a buffer against phenotypic variation, allowing genotypic variation to build. When the buffering capacity of Hsp90 is altered (e.g., in nature, by mutation or environmental stress), the genetic variation is "revealed," manifesting itself as phenotypic variation. This phenomenon raises questions about the genetic variation before and after what I will call a "revelation event": Is it neutral, nearly neutral, or non-neutral (i.e., strongly deleterious or strongly advantageous)? Moreover, what kinds of evolutionary processes do we take to be at work? Rutherford and Lindquist (1998) focus on the implications of non-neutral variation and selection. Later work by Queitsch, Sangster, and Lindquist (2002) and Sangster, Lindquist, and Queitsch (2004) raises the possibility that Hsp90 
buffering may play the role that was played by drift in sewall Wright's shifting balance model, permitting transition from one adaptive peak to another. However, Ohta (2002) suggests that much of this variation may be nearly neutral, which in turn, would imply a strong role for drift as well as selection. The primary goal of this paper is to illuminate the alternative scenarios and the processes operating in each. At the end, I raise the possibility of a synthesis between evo-devo and nearly neutral evolution.

\section{Keywords}

adaptationist, developmental buffer, evo-devo, evolution, evolvability, heat shock protein, Hsp90, natural selection, nearly neutral theory, random genetic drift 
In 1998, Suzanne Rutherford and Susan Lindquist's study of the heat shock protein known as Hsp90 was published in Nature. The paper quickly became well cited among biologists and philosophers who study evolutionary developmental biology, or evo-devo (see, e.g., Gilbert 2000; Hall 2001; Pigliucci 2002; Kaplan forthcoming). In particular, the study has been included among the pieces of evidence taken to show the essential role of developmental processes in evolution; Hsp90 acts as a buffer against new mutations, allowing for "normal" development while underlying genotypic variation increases. When the buffering capacity of Hsp90 is altered (e.g., in nature, by mutation or environmental stress), the genetic variation is "revealed," manifesting itself as phenotypic variation.

So, on the face of it, it would seem that this developmental phenomenon must have evolutionary consequences, but what are those evolutionary consequences? Some brief suggestions have been made, stemming from concerns that arise out of developmental biology as well as population genetics and molecular evolution. In particular, it has been suggested that developmental buffering can give rise to adaptive morphological evolution, to shifts among "adaptive peaks," to the adaptive radiation of lineages - and, provocatively, to nearly neutral evolution. It has also been suggested that Hsp90 is part of a selected mechanism for evolvability. However, in order to understand and evaluate these suggestions, we must first identify the stages that populations would go through (beginning with developmental buffering and ending with evolutionary change) and the processes involved at each stage; these stages and processes have not been described 
explicitly by the authors involved. Once that is done, the suggestions can be developed into full-fledged scenarios, describing the processes that would be operating at each stage under each scenario. It turns out that while the proposals have much in common, they do disagree on some key claims. Thus, one goal of the present paper is to identify and characterize alternative scenarios and the various processes that would be operating under each. Issues also arise with respect to the way that evo-devo claims about Hsp90 fit into larger discussions over developmental biology's relationship to the 20 th century evolutionary synthesis. For example, Amundson (2005) depicts two competing approaches, which he calls structuralist (developmental) and functionalist (neo-Darwinian), the latter being the approach of the synthesis. He also contrasts these two approaches by referring to the former as structuralist and the latter as adaptationist. However, I don't think it is appropriate to label only the second approach as "adaptationist." ${ }^{1}$ As will quickly become evident from my discussion below, there is good reason to think that the developmentalist project is adaptationist as well.

of course, this raises the question of exactly what is "evo-devo" or "devo-evo," an interesting and worthwhile discussion that would take us far afield, so I will not discuss it here (but see, e.g., Hall 2000). Thus, the risk of my claim is that the aspects I am describing as adaptationist will not be seen as part of either evo-devo or devoevo. So, I will make only the minimal claim that some of the claims

\footnotetext{
${ }^{1}$ Even though there are genuine tensions between the two approaches, as Amundson clearly and carefully describes.
} 
made by those who are interested in some of the intersections of evolution and development are adaptationist, although the stronger claim may be justified, too; see, e.g., von Dassow and Munro (1999). In any case, I will continue to use the convenient term "evo-devo." Finally, I will offer some thoughts on the significance of the fact that there are alternative scenarios and where they might take us in the future. The study of Hsp90 is a case where three areas of biology - population genetics, molecular evolution, and evo-devo bump into each other. This raises the potential for conflict, but it also raises the potential for synthesis: a synthesis between the nearly neutral theory and evo-devo. My hope is to have laid a bit of the groundwork for such a synthesis, should it prove desirable and feasible.

\section{Hsp90 as a Developmental Buffer: Experimental Results}

I will begin by briefly summarizing Rutherford and Lindquist's (1998) study of the heat shock protein known as Hsp90, "one of the most abundant cytosolic proteins in eukaryotes" (Sangster et al. 2004: 349).2 Rutherford and Lindquist reduced Hsp90 production in Drosophila by three means: 1) pharmacologically, in the laboratory (they fed the fruit flies food which contained a potent, specific

2 This is not exactly true, strictly speaking; Sangster et al. state: “The molecular weights and names of Hsp90 homologs differ between organisms but, for simplicity, we refer to all as Hsp90. The essential function of Hsp90 is conserved among eukarya" (2004: 349). 
inhibitor of Hsp90), 2) by breeding mutants that are heterozygous at the Hsp83 locus ${ }^{3}$ (homozygous mutants do not survive), and 3 ) by exposing them to high temperatures. They found that lowering Hsp90 production led to a wide variety of developmental abnormalities, including "body-part transformations, disrupted abdominal patterning, bristle duplications, deformed eyes or legs and changes in wing shape or venation" (Rutherford and Lindquist 1998: 336).

Similar results were produced by Christine Queitsch, Todd Sangster, and Lindquist in a subsequent study of the flowering plant Arabidopsis thaliana (2002). Queitsch et al. varied the concentration of Hsp90 and found that whereas low concentrations of Hsp90 merely yielded multiple phenotypes with reduced viability, at slightly higher concentrations, "most plants remained healthy and unaffected but some exhibited strongly altered phenotypes" (2002: 619). Again, a variety of characteristics were affected: shape; color and expansion of cotyledons; shape, color, and presence of true leaves; shape and length of hypocotyls; root morphology; and the orientation of rosettes, roots, or whole seedlings.

Given that normal development is disrupted when less Hsp90 is available to the organism, the Drosophila and Arabidopsis results, taken together, suggest that Hsp90 plays a significant role in development. They also suggest that the phenomenon may be widespread, given that the results were produced in evolutionarily distant lineages, and that Hsp90 is one of the most abundant cytosolic proteins in eukaryotes.

${ }^{3}$ Hsp83 is a homolog of Hsp90 (see previous footnote). 
Rutherford and Lindquist consider "three possibilities of increasing interest" to explain the disruption to normal development in Hsp90 mutants: 1) Wild-type Hsp90 fruit flies “might simply buffer against 'developmental noise' caused by random micro-environmental effects" (2002: 337). The mutants would lack this capability, but the developmental abnormalities would not have any other underlying genetic basis. 2) “Hsp90 mutants might exhibit an increased mutation rate," which would occur if Hsp90 were directly or indirectly involved in the fidelity of DNA replication" (2002: 337). 3) Because Hsp90 is a chaperone for signal-transduction elements, it might normally suppress the expression of genetic variation affecting many development pathways, so that when Hsp90 is less available for suppression, the previously hidden variation might be expressed to a greater extent. Rutherford and Lindquist maintain that their evidence supports the third possibility; subsequent crosses revealed defects that were specific to particular stocks, suggesting that there really was hidden genetic variation, exhibited in patterns that were unlikely to have been produced by de novo mutation.

Rutherford and Lindquist argue that under normal conditions, Hsp90 assists in protein folding during development, so that not all genetic variation is expressed as phenotypic variation. Thus, Hsp90 acts as a developmental "buffer" against genetic mutations, maintaining normal development. 4 When it is present in sufficient

4 Hsp90 does this through repeated cycles of binding and release to "signal transducers" involved in cell cycles and developmental regulation. These signal transducer proteins are “inherently 
quantities, genetic variation can accumulate. However, when it is reduced, the genetic variation is revealed as phenotypic variation. One of the things that can reduce the amount of Hsp9o available to an organism is environmental stress. Rutherford and Lindquist suggest that Hsp90 plays a dual role: it is (by their arguments) a developmental buffer as well as a mechanism through which an organism responds to environmental stress. This dual role raises the possibility of a link between environmental contingencies and developmental programs, a link with potentially important evolutionary consequences in natural populations. (Of course, any other stress protein which played a similar dual role might have similar evolutionary consequences). To show how this would work in nature, I identify and characterize four distinct evolutionary phases: 1) buffering, 2) culling, 3) establishing a foothold, and 4) transforming.

\section{Four Phases of Hsp90-Induced Evolution}

In the buffering phase, during "normal" times where Hsp90 is present, genetic variation would accumulate in the population. Since the

unstable"; Hsp90 stabilizes the tertiary structure of the proteins, permitting normal cellular and developmental signaling processes to be maintained. (It is a "chaperone" for signal-transduction elements). So, reduced Hsp90 production manifests itself as developmental abnormalities. 
genetic variation would be phenotypically silent, it would be neutral 5 with respect to selection, and therefore subject to random drift. 6 Given random drift, the frequencies of the genetic variants would fluctuate from one generation to the next; by chance, some of the genetic variants might increase in frequency whereas others might be eliminated accidentally. Then, when the population was subject to a severe environmental stressor (e.g., extreme temperatures), Hsp90 would be diverted to aid in the folding of stress-damaged proteins, making less available for developmental buffering. This would cause the genotypic variation to be revealed as phenotypic variation (i.e., there would be what I will call a revelation event). The revelation event may give rise to a culling phase, where deleterious variants would be quickly eliminated through natural

5 It is possible that some of the buffered variants would vary slightly in fitness even though they are not being expressed phenotypically, e.g., if some incurred a slight cost to the organism. In this case, the variants would be nearly neutral and subject to weak selection outweighed by drift. Nearly neutral variants will be discussed further below.

6 Here I use the term 'random drift' to refer to the indiscriminate sampling process whereby heritable physical differences between entities (e.g., organisms, gametes) are causally irrelevant to differences in reproductive success. Other authors define 'drift' in terms of its outcome rather than as a process, but this raises difficulties (see Millstein 2002, 2005 for discussion). 
selection. Rutherford and Lindquist's results suggest that in Drosophila, most of the newly uncovered phenotypic variation will be strongly deleterious. On the other hand, Queitsch et al.'s results with Arabidopsis seem to show fewer deleterious abnormalities to be eliminated by selection. Regardless of the numbers, however, the culling process would be the same.

In the establishing a foothold phase, the phenotypic traits would appear even in the presence of Hsp90 so that the traits could be subject to further evolution even after the environmental stress had ended, when Hsp90 levels were raised again. 7 Rutherford and Lindquist suggest that if the traits in question were polygenic "threshold" traits, requiring a minimum number of genes for expression, and if Hsp90 "lowers" the threshold for expression of the trait, then selection on the trait could increase the frequency of these alleles in the population, increasing the chances of being coupled with other alleles for the same trait. Their results confirmed that several generations of selection permitted expression of the traits even after Hsp90 levels were raised. However, since drift can lead to chance increases in gene frequency, it too could be the means by which traits "establish a foothold."

This creates the possibility for a transforming phase, a phase where evolutionary processes act on the remaining phenotypic variation (i.e., the genetic variation that was not eliminated in the second phase). Indeed, it raises the possibility that the revelation event 7 This will be discussed further below, in the context of discussing genetic assimilation. 
due first to the presence of Hsp90 developmental buffering and then to the absence of Hsp90 developmental buffering - could have long-term evolutionary consequences.

The nature of these evolutionary consequences, however, depends on two interconnected questions. First, is the revealed variation selectively advantageous, selectively disadvantageous, or selectively neutral? And second, what processes will be acting on that variation in the third evolutionary phase? In particular, will it be primarily selection, primarily random drift, some combination of selection and drift, or additional developmental processes?

\section{Three Scenarios for the Evo-Devo Implications of Hsp90}

I identify three possible scenarios for the evolutionary developmental implications of Hsp90: 1) selectionist/adaptationist, 2) (nearly) neutral, 3) developmental. These scenarios are not mutually exclusive, and I imagine they are not exhaustive either, but my hope is that this classification will prove useful. To clarify the starting point of my analysis, I am assuming that each of these possible scenarios agrees on the occurrence of the buffering, culling, and establishing a foothold phases (although as will be discussed further below, some of the scenarios differ in the way that they highlight various aspects of these phases). Where the different positions primarily diverge is with respect to the transforming phase, after the genetic variation has been revealed as phenotypic variation, after the strongly deleterious mutations have been eliminated, and 
after the phenotypic variation appears even in the presence of Hsp90. That is, the question at hand concerns the remaining "footholded" genetic variation of the transforming phase. This is not to suggest that the buffering (pure random drift) phase is evolutionarily unimportant. As Sangster et al. note,

This effect of the Hsp90 buffer would have the greatest impact when the desirable allele is at low frequency and most susceptible to loss from the population by genetic drift. Because of drift, even advantageous alleles may need to arise independently several times before becoming established. Each mutation is a rare event; thus, an increase in penetrance of recessive alleles may dramatically hasten their fixation and speed phenotypic evolution (Sangster et al. 2004: $356)$.

What Sangster et al. seem to be suggesting here is that the developmental buffering provided by Hsp90 allows time for mutations to arise more than once, providing a "critical mass" for selection to act upon in the transforming phase. However, Sangster et al seem to be overlooking the fact drift is acting during the developmental buffering. Furthermore, drift may help or hinder (or leave essentially unchanged) the accumulation of mutations that arise in the buffering phase, depending on the direction of the random fluctuations. And clearly, the establishing a foothold phase is evolutionarily important as well, as will be discussed further below.

\section{Scenario 1: The Selectionist/Adaptationist Scenario}


As we will see, there are actually three versions of the first scenario, the selectionist/adaptationist scenario, that vary in the degree of adaptation proposed. Rutherford and Lindquist refer to Hsp90 as a "capacitor for morphological evolution" and suggest that during "normal" times, Hsp90 would act like an electric capacitor, 8 allowing unexpressed genetic variation to accumulate temporarily. Then, during times of environmental stress, the reduction in Hsp90 available for developmental buffering "could uncover morphological variants for selection to act upon" (Rutherford and Lindquist 1998: $341)$.

So, the most minimal version of the selectionist/adaptationist scenario is simply the claim that in the transforming phase, some of the revealed genetic variation provides an advantage to some of the organisms in the population, so that selection can act upon it. The result would be adaptive morphological evolution. Rutherford and Lindquist acknowledge that other models and experiments have similarly highlighted developmental homeostasis and its disruption; however, they claim, "both the wide variety and unusual character of the morphological variation uncovered when Hsp90 is impaired, and the prevalence of natural stresses that might disrupt it, are unprecedented" (Rutherford and Lindquist 1998: 341) .

8 A capacitor is an electric circuit element, present in virtually every piece of electronic equipment in use today. It functions to accumulate, and temporarily hold, electric charge. The charge is later returned to the circuit. 
More strongly, Rutherford and Lindquist speculate, "The use of Hsp90 as a capacitor for the conditional release of stores of hidden morphogenic variation may have been adaptive for particular lineages, perhaps allowing the rapid morphological radiations that are found in the fossil record" (Rutherford and Lindquist 1998: 341). In other words, assuming that some of the revealed variation is beneficial to the organisms in the population, revelation events may not only spur selection in an adaptive direction; they may also spur adaptive radiation of entire lineages. This claim is clearly spurred by the wide variety of morphological variation that was uncovered; these are more than trivial variations.

This yields two versions of the selectionist/adaptationist scenario: 1.1) Hsp90 could act as a capacitor for adaptive morphological evolution and 1.2) Hsp90 could be responsible for the rapid (and presumably adaptive) morphological radiations seen in the fossil record

But there is a third selectionist/adaptationist scenario at hand, brought about by Sangster et al.'s attempt to solve a "classic evolutionary dilemma" - how can populations shift from one "adaptive peak" to another if the intermediate states have reduced fitness? The shifting balance model was Sewall Wright's solution to the problem of transitions between adaptive peaks. According to Wright, if populations were subdivided into smaller subpopulations, then slightly deleterious alleles could accumulate by drift. This would permit the 
transition from one adaptive peak to another. 9 But Sangster et al. suggest that Hsp90 buffering, in allowing the accumulation of deleterious alleles, could also facilitate the transition from one adaptive peak to another. 10 Thus, Hsp90 could play the same role that subdivided populations play in Wright's shifting balance theory. In fact, its role might even be more significant, Sangster et al. imply, given that it is a "genome-wide" phenomenon induced by stressful conditions.

However, note that (if my analysis is correct here), drift is playing a role in either case; what is different is that in Wright's scenario, drift is occurring in subdivided populations, whereas under Sangster et al.'s proposal, drift is occurring as a consequence of the Hsp90's developmental buffering. It is also important to note that the developmental buffering ultimately gives rise to adaptive evolution (adaptive evolution having been the outcome of Wright's shifting balance model as well). So, this gives us a third version of the selectionist/adaptationist scenario (1.3), since the transforming phase is, once again, a selection phase yielding adaptive evolution. One final point of clarification is in order with regard to this scenario. My reason for labelling it as "selectionist/adaptationist" is not that I think the authors discussed above are making claims

9 I am leaving out the details of Wright's own three phase model since they are not relevant to us here; see Skipper (2002) for discussion. 10 The same set of authors gives a similar discussion in Queitsch et al. (2002) . 
concerning the prevalence of selection and adaptation. Rather, my point is that they are being selectionist and adaptationist in a much more minimal sense: all three versions described invoke only selectionist and adaptationist explanations. No doubt, all the authors would admit the existence of alternative explanations. However, as Stephen Jay Gould and Richard Lewontin note in their critique of the so-called "adaptationist programme": "The admission of alternatives in principle does not imply their consideration in daily practice" (1979: 586). What I am commenting on here is exactly that the authors' lack of consideration of alternatives. (Issues surrounding adaptationism will be discussed further below).

\section{Scenario 2: The (Nearly) Neutral Scenario}

The second scenario I will discuss has been suggested by Tomoko Ohta. Before describing this alternative, however, it will be helpful to briefly describe Ohta's nearly neutral model. (Ohta has modified the nearly neutral model over the years; my account will be limited to its most recent incarnation).11 Ohta's nearly neutral model is a modification of Motoo Kimura's neutral model. Both of these models were originally developed as models of molecular evolution. That is, they were developed to account for nucleotide substitutions, or sometimes, amino acid substitutions. According to Kimura and Ohta, most new mutations are deleterious and are eliminated quickly through negative selection. 12 The question then becomes, what do we say about

11 See Dietrich and Millstein (forthcoming) for further discussion.

12 This is analogous to the culling phase I identified above. 
the remaining observed variation? Although these issues have now become controversial, it seemed initially to be the case that there were nucleotide substitutions that were neutral; they would yield the same amino acid and thus the same protein. And it was thought that amino acid substitutions could also be neutral if they yielded a functionally equivalent protein. Kimura argued that his model - a model where much of the observed variation was neutral, and therefore subject to random drift - was a better fit for the observed data than selectionist models (Dietrich 2006).

Ohta, however, argued that an even better fit could be obtained by expanding the classes of variants that are incorporated into the models. According to Ohta (2002), "selection theory" contains two classes of new mutations, deleterious and advantageous, whereas the "neutral theory" expands the borderline between these two classes to include a third class, the neutral class. Ohta argues that the "nearly neutral theory" shows that we ought to expand the borderline between deleterious and neutral and the borderline between advantageous and neutral to allow for two more classes of new mutants: weakly deleterious and weakly advantageous. As Michael Dietrich and I argue in a forthcoming paper, the nearly neutral theory should be understood as claiming that two processes are acting on these "nearly neutral" variants: the process of drift and the process of selection. However, since the nearly neutral variants are weakly advantageous or weakly deleterious, selection is likewise weak; the effects of drift thus swamp it. 
Again, both the neutral and the nearly neutral model were developed as models of molecular evolution. Although there have been hints that the models could be applied to morphological (or, at least phenotypic) evolution as well,13 recently, Ohta (2002) has argued more explicitly for applying the nearly neutral model to morphological evolution. And, in this context, she cites Rutherford and Lindquist's results as evidence for the nearly neutral theory. Her claim seems to be that, in what I have called the transforming phase, most of the revealed variation will be nearly neutral. Thus, according to the nearly neutral scenario (scenario 2.1 ), the revealed variants will be subject to weak selection and drift, with the effects of drift outweighing the effects of selection. This contrasts with the first set of scenarios, where the variants were seen as advantageous and subject to selection. And on Ohta's scenario, the resulting evolution would be nonadaptive rather than adaptive.

of course, there is a logically possible variant of this scenario in which the transforming phase variants are largely neutral (rather than nearly neutral), and thus subject to random drift alone. I'll refer to this as scenario 2.2. Although I am not aware of anyone who has advocated this scenario, I mention it for the sake of completeness. This would clearly also be a case of nonadaptive evolution.

\section{Scenario 3: Developmental Scenario}

13 See, e.g., King and Jukes' discussion of the ability to synthesize vitamin $\mathrm{C}$. 
The first two scenarios focused on evolutionary phenomena, but there are also scenarios that emphasize developmental phenomena. The four phases incorporate developmental phenomena long discussed by biologists and promise to reveal additional phenomena as well. Consider, for example, canalization. Gilbert, citing Waddington (1940), defines canalization as, "the property of developmental pathways to produce standard phenotypes despite mild environmental or genetic perturbations" (2000: 731). Canalization occurs during the buffering phase, when Hsp90 acts as a chaperone and permits "normal" cellular and developmental signaling processes to be maintained, yielding "standard" genotypes. The point here is not that the other scenarios fail to incorporate canalization - in fact, they would all acknowledge that Hsp90 helps to stabilize development - but rather that a developmental scenario would highlight the necessary role of canalization to the entire process and be able to draw connections to other instances of canalization. I'll refer to this as scenario 3.1 . Developmental scenarios for the four phases might also highlight the role of genetic assimilation (scenario 3.2). Waddington (1961) characterizes genetic assimilation as "a process by which a phenotypic character, which initially is produced only in response to some environmental influence, becomes, through a process of selection, taken over by the genotype, so that it is found even in the absence of the environmental influence which had at first been necessary." As noted previously, Rutherford and Lindquist found (during what I have called the "establishing a foothold" phase) that 80-90\% of the phenotypic variation that was initially produced only in response to 
decreased Hsp90 (as might occur when the organisms were under environmental stress), was, after several generations of selection, found even in the absence of decreased amounts of Hsp90 (i.e., even in the presence of normal amounts of Hsp90). The phenotypic response has been "taken over by the genotype." Although not solely a developmental process, since selection plays a crucial role, genetic assimilation involves developmental processes in two ways: 1) the developmental buffering phase is crucial for setting up the conditions for genetic assimilation by allowing the accumulation of genetic variation, and 2 ) it is only through a developmental processes that the genetic variation is revealed as phenotypic variation; the diverting of Hsp90 to deal with the environmental stress causes organisms to develop differently than they would have in the presence of Hsp90. Thus, it can be argued that Rutherford and Lindquist's experiments demonstrated the developmental phenomenon of genetic assimilation; Gilbert (2000) and Sangster et al (2004), among others, make this claim.

It is interesting to note, however, that in discussing the significance of genetic assimilation via Hsp90,14 Gilbert states: "Most of these morphological variations would probably be deleterious, but some might be selected for in the new environment. Such releasing of hidden morphological variation may be responsible for the radiations found in the fossil record" (2000: 733). Thus, Gilbert 14 See West-Eberhard (2003: 157) for an argument that environmentally induced traits have more evolutionary potential than mutationally induced ones. 
describes the significance of this developmental phenomenon in terms of its contribution to adaptive morphological evolution. Earlier, I labeled contribution to adaptive radiation among lineages as scenario 1.2; it was one of the scenarios that Rutherford and Lindquist had proposed. I have placed genetic assimilation in a separate section because I think it is important to highlight this developmental phenomenon, which may have consequences other than those that Gilbert describes in his 2000 essay. However, in this instance, we are looking at the same biological phenomenon as before, but from a different perspective.

Queitsch et al. (2002) propose another possible developmental scenario, again for the "establishing a foothold" phase. I'll refer to this as scenario 3.3. They suggest that,

even in the absence of stress, some populations may be so close to the trait expression threshold that stochastic events in development will produce a few individuals displaying the altered trait. Once the pathway is diverted, the expression of a new trait may become robust through the influence of auto-regulatory feedback loops, selfperpetuating protein conformations, and developmental windows (2002: $623)$.

Of course, other developmental scenarios are possible. However, I will not discuss them further in this essay. 
See Table 1 for a summary of the three scenarios and the proposed processes and outcomes ${ }^{15}$ that would occur during each phase, under each scenario.

\section{[ Insert table 1 about here ]}

\section{Mechanism for Evolvability?}

The three scenarios described above all assume that Hsp90 has already evolved, focusing instead on the consequences of Hsp90 given its presence in organisms. But they make no claim as to how it evolved. Nonetheless, the evolutionary origin of Hsp90 has played an important role in discussions over its evolutionary implications. In order to discuss this issue, we must distinguish between the various metaphors used to describe Hsp90's functions: it is said to be a "chaperone," a “developmental buffer," and a “capacitor." As a chaperone, Hsp90 stabilizes the tertiary structure of signal transducer proteins. This chaperoning permits buffering, i.e., it permits "normal" cellular and developmental signaling processes to be maintained. Buffering, which simply "stores" genetic variation, combined with environmental stress, which "releases" genetic variation, yields capacitance (defined as storage and release). We can now rephrase the issue at hand as a

15 It is crucially important to distinguish between "process" and "outcome" when discussing evolutionary phenomena (Millstein 2006). 
question: which, if any, of these three Hsp90 functions is the product of natural selection? 16

It is reasonable, and perhaps uncontroversial, to think that Hsp90's ability to chaperone is a product of natural selection; Sangster et. al (2004: 355) explicitly endorse this view. And buffering seems almost as, if not equally, unproblematic. Certainly, it could be advantageous for organisms to be able to buffer against mutations during development, given that many mutations are deleterious. Moreover, selection favoring buffering ability seems to raise no more concerns than selection favoring any other trait.17

What is controversial, however, is the claim that capacitance is a product of natural selection. The thinking goes as follows: capacitance itself could be seen as a beneficial response to stressful conditions, since it would permit the species to be flexible in its responses to the stress. Thus, capacitance could have been

16 In exchanging the term "natural selection" for "evolution," I am not suggesting that the two are equivalent. Rather, I am assuming that all of these functions have evolved in some fashion, and seek to ask the more specific question as to whether they have evolved by natural selection.

17 Sangster et al. (2004) say that they are questioning whether buffering is the product of selection. However, since they refer to buffering followed by environmental stress, it seems clear that their remarks are addressed to capacitance, not buffering. I will assume this in the text that follows. 
selectively favored, giving rise to a "mechanism for 'evolvability'"18 which Rutherford and Lindquist describe as "an explicit molecular mechanism that assists the process of evolutionary change in response to the environment" (Rutherford and Lindquist 1998: 341). The four stages described in the previous section characterize the processes underlying capacitance, with the different scenarios providing alternative versions of some of the stages. However, the term "capacitor," like many metaphors, can both elucidate and obfuscate. Rutherford and Lindquist assert that Hsp90 is a capacitor, but it would be more accurate to say that Hsp90 creates a capacitor, since Hsp90 is not itself storing anything. Rather, Hsp90 facilitates storage and release. But this yields another unclarity: what is the storage and release for? In short, what is the capacitor for, and which entity is the capacitor?

If the claim is that there is capacitance for variation, then the capacitor could be either individual organisms or individual populations. That is, when Hsp90 is present, over time individual organisms will obtain an ever-increasing store of genetic variation, and consequently, so will the populations that are composed of those organisms. However, if the claim is that there is capacitance as a mechanism of evolvability, then this applies to populations only, 18 The term "evolvability" deserves more analysis than I can give it here; for discussion, see, e.g., Dawkins 1989, Love 2003, A. Wagner 2005ab. Here I will simply express a concern that what many biologists and philosophers call "evolvability" would be more accurately called "selectability." 
since organisms do not evolve, only populations. In other words, there must be storage and release of variations in a population in order to enhance a population's evolvability. It seems as though it is this latter, and stronger claim, which is intended; it amounts to the claim that increased variation in a population as a response to environmental stress enhances the population's ability to evolve, and the claim that this enhanced ability to evolve under stressful conditions is a product of natural selection.

However, Rutherford and Lindquist have since parted ways on these claims. Whereas Rutherford (2003) has sought to strengthen the case for evolvability, Lindquist, in another co-authored paper with Sangster and Queitsch, has distanced herself from it. Rutherford notes that "[e]volvability could have arisen either through selection for its benefits to small or highly structured populations or lineages - group selection" although she acknowledges that evolvability could be "an unselected consequence of adaptive or neutral traits in individuals" (2003: 264).19 Sangster et al., on the other hand, view a mechanism for evolvability as "theoretically unlikely... since it would produce no immediately selectable phenotype" (Sangster et al. 2004: 355).20 Rather than having been a direct target of selection,

19 The former assertion makes clear that evolvability is a property of populations, as discussed in the previous paragraph; the latter assertion is more ambiguous.

20 Sangster et al. do not elaborate, but perhaps (emphasizing the term "immediately" in the quote above) their concern is that the benefit of 
they suggest that Hsp90's capacitance could be a 'spandrel' (Sangster et al. 2004: 355) - i.e., an accidental evolutionary byproduct of chaperoning.

I will not attempt to decide between these alternative proposals, 21 but I will discuss some ways in which we might shed light on them (see also Wagner et al. 1999). Mike Dietrich (personal communication, 3 October 2007) has suggested to me that, since capacitance involves presence and then absence of Hsp90, the amount of Hsp90 would be under selection. Too much Hsp90, and there would be buffering without capacitance. Following a related suggestion made by Partridge and Barton (2000), one could look for correlations between the amount of Hsp90 and stressful environments in nature; in this case, the stressfulness of the environment for the organisms in question would be inversely proportional to the amount of Hsp90 produced. However, the amount of Hsp90 is going to be bounded in any case, for two reasons: one, an overabundance of Hsp90 is detrimental to organisms in other ways, e.g., by slowing development (Sangster et al. 2004: 355); and two, (as will be discussed further below) without sufficient Hsp90 buffering never occurs. A second suggestion for

variations produced in a time of stress only occurs long after the buffering has taken place.

21 Another possible alternative: Hsp90 capacitance could have evolved in accordance with Masatoshi Nei's mutationism, where “the production of functionally more efficient genotypes by mutation... and recombination" is "the most fundamental process for adaptive evolution" (Takahata 2007: 4-5). 
deciding between the proposals, again inspired by Partridge and Barton (2000), would be to have organisms with varying amounts of Hsp90 compete in the laboratory in both constant and varied environments. These would be worthwhile investigations to undertake, although, it should be noted, they would suggest only that capacitance is maintained by selection, not that it originally evolved by selection (although the former makes the latter more plausible).

Again, let me reemphasize that the three scenarios described in the preceding section are independent of how capacitance evolved; they only describe possibilities given that capacitance has evolved. I also note that debates over adaptationism have once again resurfaced.

\section{Discussion}

There is no doubt that if Rutherford and Lindquist's results are sound, that the developmental buffering capabilities of Hsp90 are potentially quite significant evolutionarily. The confirmation of Rutherford and Lindquist's results in an evolutionary distant lineage (Arabidopsis), coupled with the ubiquity of Hsp90 and heat shock proteins in general, makes this even more likely. But in what way(s) are they evolutionarily significant? As described by the selectionist/adaptationist scenario? The neutral or nearly neutral scenario? The developmental scenario? I will now offer some thoughts about the implications of these three scenarios, some of which will be a bit speculative. 
Importantly, these scenarios are not mutually exclusive. It could be the case that processes described in all of the scenarios are operating simultaneously. Perhaps in the first "buffering" phase, canalization occurs (scenario 3.1) while drift permits shifting to new adaptive peaks (scenario 1.3 ). Then, during the during the "establishing a foothold" phase, perhaps genetic assimilation (scenario 3.2) and stochastic events in development (scenario 3.3) are taking place. And perhaps in the third "transforming" phase, selection acts on strongly advantageous variants (scenario 1.1). This selection leads to the splitting of lineages (scenario 1.2). Meanwhile, drift is acting on the neutral variants (scenario 2.2 ); weak selection outweighed by drift is acting on the nearly neutral ones (scenario $2.1) \cdot$

That all of these processes are occurring simultaneously in nature may be, in fact, the most likely scenario. In short, I don't mean to present these scenarios as though there is a debate going on. There isn't. (Or, at least there isn't yet). Ohta's citation of Rutherford and Lindquist's results gives no indication that she is taking issue with any of their claims. Rather, she cites their results as one more piece of evidence in support of the nearly neutral theory. On the other hand, as far as I can determine, Ohta's paper has not been cited by those authors more interested in the developmental and adaptive consequences of Hsp90. If there were to be a debate, clearly one point of disagreement would be between the neutral/nearly neutral scenario and the other 
scenarios.22 And then, this hypothetical debate would most likely take the form of a relative significance debate (Beatty 1997), with proponents arguing for the prevalence of their preferred scenario over the other scenarios, rather than taking the position that one scenario operated to the exclusion of the other scenarios. In fact, it would probably look very much like the neutralist/selectionist debates (or, if you like, adaptationist/nonadaptationist debates) that have already taken place - and are taking place - within the fields of molecular evolution and phenotypic evolution. In short, biologists have been arguing over the relative prevalence of adaptive and nonadaptive characters for decades. The positions and the proposed methods of solution are well staked out. Although there could be something new, it is unlikely that this new debate, were it to occur, would cover any new ground, or be any more likely to be resolved, given the seemingly unending nature of its predecessors.

Instead, we might ask why different authors have proposed different scenarios. A first pass at answering that question might be to suggest that biologists are operating under their preexisting paradigms. Ohta has long argued for the prevalence of nearly neutral variants; perhaps it is no surprise, then, that she would be attentive to the possibility of nearly neutral variants among the variation revealed subsequent to Hsp90 buffering. However, it is a bit more puzzling that those arguing for the essential role of developmental processes in evolution would set those processes only in an adaptive

22 Another, perhaps, might be between the developmental scenarios and the more evolutionary-oriented scenarios. 
context, especially given Amundson's claim, mentioned at the outset of my paper, that developmental approaches are in tension with adaptationist approaches. (Of course, it is unsurprising that they would seek out further developmental consequences of those phenomena). Indeed, I find it somewhat ironic that people who are otherwise unorthodox in their thinking with respect to evolution are so orthodox when it comes to adaptationism. After all, as the late Gould argued, nonadaptive approaches were left out of the evolutionary synthesis (Gould 1983) just as developmental processes were (Gould 2002).

A second pass at answering the question of why different authors have proposed different scenarios might be that the various scenarios serve as legitimization for their respective claims. Rutherford and Lindquist are able to show that their Hsp90 results are significant by tying them to adaptive evolution, perhaps even on a macroevolutionary scale. (Even the scenario that looks like Wrightian shifting balance is, as noted previously, in the service of adaptive evolution). Here the implication is that those evolutionary consequences are important only if they are adaptive. And yet, I am inclined to agree with Godfrey-Smith (2001) that this form of adaptationism (what GodfreySmith dubs "explanatory adaptationism") is really just a personal preference regarding what is taken to be interesting or not. One might equally well take Kimura's view that "what is important in science is to find the truth, so the neutral theory should be of value if it is valid as a scientific hypothesis" even if "neutral genes are by definition not concerned with adaptation" (1983: 325). 
However, let us set those concerns aside. Despite continuing debates, it is probably fair to say that the evolutionary synthesis (for better or for worse) is largely an adaptationist one. Greater legitimization can be achieved if one can show that one's phenomenon fits into that larger picture. But this sounds far more Machiavellian than I intend it to; I am merely stating a fact, not implying any deliberate intention on the part of the authors. Showing that Hsp90 may have important consequences for adaptive evolution is a significant result indeed.

Whereas Rutherford and Lindquist's data can be legitimated by showing its evolutionary implications, for Ohta the situation works in reverse; her evolutionary claims can be legitimated by showing how they can fit Rutherford and Lindquist's data. That is, the suggestion is that Rutherford and Lindquist's results provide additional reasons (reasons other than the many others which Ohta has proposed) to think that nearly neutral variants are prevalent in nature. Other than showing their possible consistency with the data, however, she does not give us any reason to think that much of the revealed variation is in fact nearly neutral. Still, however, a form of legitimization is achieved by Ohta, and certainly the new results do not overturn the nearly neutral model.

I should be clear, though, that at this point neither "side" has given us much reason to think that most (or much) of the revealed variation is advantageous or that most (or much) of the revealed variation is nearly neutral; all we have are speculations. Moreover, little data has been presented that comes even close to resolving this 
question. All we have are the laboratory data with Drosophila and Arabidopsis. Queitsch et al. (2002) acknowledge that “it is difficult to predict what might prove adaptive in evolution," but they argue,

phenotypes revealed in plants by challenging Hsp90 buffering capacity are not 'monstrous' in character (as they might be described in fruitflies). Indeed, some would seem plausibly advantageous under particular conditions: for example, altered leaf shapes, purple pigment accumulation, and different degrees of hypocotyl extension (2002: 623).

Still, this is far from definitive, as the authors seem to concede. The results suggest that the revealed variations of organisms are sensitive to the amount of Hsp90 available for developmental buffering, so the variations produced would depend on the degree of environmental stress. And then, of course, whether the variations were advantageous, deleterious, or somewhere in between, would depend on the new environmental conditions and how long they persisted. These types of data are not easy to obtain. Again, these issues put us right back into the same old set of problems in deciding claims concerning adaptation.

Frédéric Bouchard (personal communication, 26 July 2007) has suggested to me that the balance of processes might be different in Arabidopsis and Drosophila. Perhaps, for example, selectionist/adaptationist scenarios prevail in Drosophila, given the severity of the changes produced, whereas a neutral/nearly neutral scenario is more appropriate for Arabidopsis, where the changes were much smaller. Moreover, as Francesca Merlin (personal communication, 
3 October 2007) has suggested to me, different scenarios might prevail for the same species at different times. Both of these suggestions may be correct, and they highlight the point that whereas adaptationist debates may be fruitless globally (Beatty 1997), they may be resolvable for more localized populations (see, e.g., Millstein forthcoming for one such case). Nonetheless, our models would still need to account for the different possible scenarios.

Thus, I wonder whether debate among the scenarios is the best outcome. Perhaps integration - synthesis - is also possible? Indeed, as I said at the outset, one of the things that interests me about this case is the collision of population genetics, molecular evolution, and evo-devo. Following Gerson (2007), true "intersections" of fields contrast with cases where there are "independent lines of research"; sometimes "bridges" among the latter can eventually yield the former. The citation pattern shows that we currently do not have a true intersection; evo-devo and the nearly neutral theory are independent lines of research both drawing from yet another line of research: biochemistry. But perhaps the Hsp90 results can serve as a bridge between the nearly neutral theory and evo-devo. Amundson (2005) is somewhat pessimistic about the possibility of a synthesis between evo-devo and population genetics. Others have been more optimistic. For example, Hall (2000) and G. Wagner (2000) imply the possibility of synthesis. G. Wagner (2007) develops the idea further. And Wimsatt and Schank (2004) develop one such model; notably, it incorporates nearly neutral mutations, although it is not a model of the particular system discussed here. However, no one (to 
my knowledge) has suggested integrating evo-devo and the nearly neutral theory.23 Yet, as Mitchell and Dietrich (2006) argue, it is pluralistic cases such as these that are the ripest for integration, i.e., cases where there can be multiple contributing causes (here, developmental processes, strong and weak selection processes, and drift processes) and/or multiple constellations of causes (e.g., selection processes predominating in some populations and drift populations prevailing in others).

What would such an integration look like? Here are some sketchy suggestions. To the extent that Rutherford and Lindquist's claims are legitimated by appeal to adaptationism, they could be equally well legitimated by an appeal to nonadaptationism. That is, the main point is to show the evolutionary consequences of the developmental buffering. However, those consequences need not be adaptive ones. In other words, the "evo" in "evo-devo" doesn't have to be adaptationist; there could be a nearly neutral theory of evo-devo. As inspiration, we can look to Gould, who, as I mentioned previously, argued for both the role of development in evolution and the importance of considering nonadaptationist approaches.

For this to work, some modifications would be needed in the population genetics/molecular evolution models as well. For example, the models would have to take into account the probability of a revelation event (which would require information concerning the 23 of course, such an integration is suggested by Ohta's own citation of the Hsp90 research. Dietrich (forthcoming) sees Ohta's work as an integrative explanation linking molecular and morphological evolution. 
environment and the developmental system itself) and the frequency of revelation events (Sangster et al. point out that the evolutionary significance of Hsp90 depends on how often stress triggers a revelation event - too often, and variation doesn't accumulate, but not often enough, and the buffered variation is never released). And they would need to account for the amount and degree of the variation produced. Integration may prove difficult, but perhaps it is not impossible.

\section{Acknowledgments}

Thanks to Mike Dietrich for the pointer to the Hsp90 research and its connections to evo-devo and the nearly neutral theory, as well as for many fruitful discussions on the neutral and nearly neutral theories in general. Thanks also to Elihu Gerson, Jim Griesemer, and Bill Wimsatt for invaluable assistance in clarifying the goals of my project, and to Mike Dietrich, John Lynch, Francesca Merlin, Ayelet Shavit, and three anonymous reviewers for helpful comments on earlier drafts. Finally, thanks to the audience members at the $2007 \mathrm{CSU}$ Northridge Philosophy of Biology Conference and at the 2007 ISHPSSB (International Society for the History, Philosophy, and Social Studies of Biology) meeting for helpful discussion on an earlier version of this paper. 


\section{REFERENCES}

Amundson, R. (2005). The changing role of the embryo in evolutionary thought. Cambridge: Cambridge University Press.

Beatty, J. (1997). Why do biologists argue like they do? Philosophy of Science 64 (Proceedings): S432-S443.

Dawkins, R. (1989). The evolution of evolvability. In C. G. Langton (Ed.), Artificial life, the proceedings of an interdisciplinary workshop on the synthesis and simulation of living. Redwood City, $\mathrm{CA}$ : Addison-Wesley •

Dietrich, M. R. (2006). Three perspectives on neutrality and drift in molecular evolution. Philosophy of Science 73: 666-677.

Dietrich, M. R. (forthcoming). Molecular evolution. In S. Sarkar, and A. Plutynski (Eds), A companion to philosophy of biology. Blackwell, Malden, MA.

Dietrich, M. R., Millstein, R. L. (forthcoming). The role of causal processes in the neutral and nearly neutral theories. Philosophy of Science.

Gerson, E. M. (2007). The juncture of evolutionary and developmental biology. In M. Laubichler \& J. Maienschein (Eds.), From embryology to evo-devo: A history of developmental evolution. Cambridge, MA: MIT Press.

Gilbert, S. F. (2000). Diachronic biology meets evo-devo: C. H. Waddington's approach to evolutionary developmental biology. American Zoologist 40: 729-737. 
Godfrey-Smith, P. (2001). Three kinds of adaptationism. In S. H.

Orzack \& E. Sober (Eds.), Adaptationism and optimality. Cambridge: Cambridge University Press.

Gould, S. J. (1983). The hardening of the modern synthesis, pp. 71-93. In M. Grene (Ed.): Dimensions of Darwinism. Cambridge University Press, Cambridge, UK.

Gould, S. J. (2002): The structure of evolutionary theory. The Belknap Press of Harvard University Press. Cambridge, MA.

Gould, S. J., and Lewontin, R. C. (1979). The spandrels of San Marco and the Panglossian paradigm: A critique of the adaptationist programme. Proceedings of the Royal Society of London B 205: 581598.

Hall, B. K. (2001). A commentary on 'Evolutionary developmental biology: Paradigms, problems and prospects.' American Zoologist $41: 1049-1051$.

Kaplan, J. (2006). Evolutionary innovations and developmental resources: From stability to variation and back again., PSA 2006 . Vancouver, BC.

Kimura, M. (1983). The neutral theory of molecular evolution. Cambridge: Cambridge University Press .

Love, A. C. (2003). Evolvability, dispositions, and intrinsicality. Philosophy of Science 70: 1015-1027.

Millstein, R. L. (2002). Are random drift and natural selection conceptually distinct? Biology and Philosophy 17(1): 33-53.

Millstein, R. L. (2005). Selection vs. drift: A response to Brandon's reply. Biology and Philosophy 20(1): 171-175. 
Millstein, R. L. (2006): Discussion of four case studies on chance in evolution: Philosophical themes and questions. Philosophy of Science $73(5): 678-687$.

Millstein, R. L. (forthcoming). Distinguishing drift and selection empirically: 'The great snail debate' of the 1950s. Journal of the History of Biology.

Mitchell, S., and Dietrich, M. R. (2006). Integration without unification: an argument for pluralism in the biological sciences. The American Naturalist 168, Supplement: S73-S79.

Ohta, T. (2002). Near-neutrality in evolution of genes and gene regulation. Proceedings of the National Academy of Sciences $99(25): 16134-16137$.

Pigliucci, M. (2002). Buffer zone. Nature 417: 598-599. Queitsch, C., Sangster, T. A., Lindquist, S. (2002). Hsp90 as a capacitor of phenotypic variation. Nature 417: 618-624. Partridge, L., and Barton, N. H. (2000). Evolving evolvability. Nature $407: 457-458$.

Rutherford, S. L. (2003). Between genotype and phenotype: Protein chaperones and evolvability. Genetics 4: 263-274.

Rutherford, S. L., Lindquist, S. (1998). Hsp90 as a capacitor for morphological evolution. Nature 396: 336-342.

Sangster, T. A., Lindquist, S., Queitsch, C. (2004). Under cover: Causes, effects and implications of hsp90-mediated genetic capacitance. Bioessays 26: 348-362.

Skipper, R. A. (2002). The persistence of the R. A. Fisher-Sewall Wright controversy • Biology and Philosophy 17: 341-367. 
Takahata, N. (2007). Molecular clock: An anti-neo-Darwinian legacy. Genetics $176: 1-6$.

von Dassow, G., Munro, E. (1999). Modularity in animal development and evolution: Elements of a conceptual framework for evodevo. Journal of Experimental Zoology 285: 307-325.

Waddington, C. H. (1961). Genetic assimilation. Advances in Genetics $10: 257-293$.

Wagner, A. (2005a). Robustness, evolvability, and neutrality. FEBS Letters 579: 1772-1778.

Wagner, A. (2005b). Robustness and evolvability in living systems. Princeton: Princeton University Press.

Wagner, G. P. (2000). What is the promise of developmental evolution? Part $i$ : Why is developmental biology necessary to explain evolutionary innovations? Journal of Experimental Zoology 288: $95-98$

Wagner, G. P. (2007). How wide and how deep is the divide between population genetics and developmental evolution? . Biology and Philosophy 22: 145-153.

Wagner, G. P., Chiu, C.-H., and Hansen, T. F. (1999). Is hsp90 a regulator of evolvability? Journal of Experimental Zoology 285: 116-118.

West-Eberhard, M. J. (2003). Developmental plasticity and evolution. Oxford: Oxford University Press.

Wimsatt, W. C., Schank, J. (2004). Generative entrenchment, modularity, and evolvability: When genetic selection meets the 
whole organism. In G. Schlosser \& G. Wagner (Eds.), Modularity in development and evolution. Chicago: University of Chicago Press. 